Chirurg 2019 90 (Suppl 2):S34 https://doi.org/10.1007/s00104-019-0903-7 Online publiziert: 13. Februar 2019

(c) Springer Medizin Verlag $\mathrm{GmbH}$, ein Teil von Springer Nature 2019

\section{Originalpublikation}

van Dijk ST, van Dijk AH, Dijkgraaf MG, Boermeester MA (2018) Meta-analysis of inhospital delay before surgery as a risk factor for complications in patients with acute appendicitis. Br J Surg 105:933-945. https:// doi.org/10.1002/bjs.10873

Hintergrund. Die akute Appendizitis gehört mit einem Lebenszeitrisiko von $7-8 \%$ zu den häufigsten chirurgischen Notfällen auf der Welt. Ursprünglich wurde angenommen, dass jede unkomplizierte Appendizitis unbehandelt zur Perforation führt. Die daraus abgeleitete Empfehlung, eine Appendizitis möglichst ohne Zeitverzögerung mit einer Appendektomie $\mathrm{zu}$ behandeln, stammt noch aus dem 19. Jahrhundert, als die perforierte Appendizitis mit einer hohen Letalität assoziiert war. Neuere Untersuchungen stellen allerdings den Übergang der unkomplizierten in die perforierte Appendizitis infrage. Die hier vorgestellte Metaanalyse untersuchte, ob eine verzögerte Appendektomie bei der unkomplizierten Appendizitis mit einem schlechteren Outcome assoziiert ist.

Methoden. In diese Metaanalyse wurden Studien aus den Jahren von 1990 bis 2016 eingeschlossen, die verschiedene Zeitintervalle zwischen Diagnosestellung und Appendektomie verglichen. Primärer Endpunkt war das Vorliegen einer komplizierten (perforierten oder

Dieser Beitrag wurde erstpubliziert in Der Chirurg (2018) 89:829-829. https://doi.org/10. 1007/s00104-018-0699-x

\title{
Metaanalyse zur verzögerten Appendektomie bei unkomplizierter Appendizitis
}

gangränösen) Appendizitis zum Zeitpunkt der Appendektomie.

Ergebnisse. Insgesamt wurden 45 Studien mit einer Gesamtzahl von 152.314 Patienten eingeschlossen. In der gepoolten Analyse zeigte sich, dass eine verzögerte Appendektomie kein signifikant höheres Risiko für das Auftreten einer komplizierten Appendizitis darstellt. Dies traf sowohl für den Vergleich der Zeiträume 0-6h vs. 7-12 h (Odds Ratio [OR] 1,07; $95 \%$-Konfidenzintervall [KI] 0,98-1,17) als auch für den Vergleich der Zeiträume $0-12 \mathrm{~h}$ vs. $13-24 \mathrm{~h}$ (OR 1,09; $95 \%$-KI 0,95-1,24) zu. Für die verzögerte Appendektomie war keine Assoziation mit einer erhöhten Morbidität nachweisbar, sofern diese innerhalb der ersten $24 \mathrm{~h}$ nach Diagnosestellung durchgeführt wurde.

Diskussion und Fazit. Diese Metaanalyse zeigt, dass eine um bis zu $24 \mathrm{~h}$ verzögerte Appendektomie zu keiner höheren Rate an komplizierter Appendizitis und $\mathrm{zu}$ keiner höheren Komplikationsrate führt. Während viele Leitlinien keine Angabe zum empfohlenen Operationszeitpunkt bei der unkomplizierten Appendizitis machen, werden von zwei bekannten Leitlinien widersprüchliche Empfehlungen ausgesprochen. Während die Leitlinie der World Society of Emergency Surgery von 2016 zum Schluss kommt, dass eine um 12-24 h verzögerte Appendektomie bei der unkomplizierten Appendizitis sicher ist [1], empfiehlt die Leitlinie der European Association of Endoscopic Surgery von 2016 eine möglichst frühzeitige Operation aufgrund des erhöhten Perforationsrisikos [2]. Auch wenn diese Metaanalyse eine große Heterogenität besitzt, stellt sie die bisher umfangreichste Arbeit zu diesem Thema dar. Sie untermauert die Hypothese, dass die unkomplizierte und komplizierte Appendizitis zwei verschiedene Entitäten mit unterschiedlicher Pathophysiologie darstellen. Die Ergebnisse der Arbeit können beispielsweise auch bei eingeschränkter nächtlicher Operationskapazität für die Indikationsstellung zur Notfalllaparoskopie berücksichtigt werden. Für die Appendizitis mit klinischen oder radiologischen Hinweisen auf einen komplizierten Verlauf (z.B. Perforation) wird nach wie vor eine möglichst frühzeitige Appendektomie empfohlen.

\section{Korrespondenzadresse}

Prof. Dr. M. Anthuber

Klinik für Allgemein-, Viszeral- und Transplantationschirurgie, Klinikum Augsburg Stenglinstr. 2, 86156 Augsburg, Deutschland matthias.anthuber@klinikum-augsburg.de

Interessenkonflikt. M. Schrempf und M. Anthuber geben an, dass kein Interessenkonflikt besteht.

\section{Literatur}

1. Di Saverio S, Birindelli A, Kelly MD et al (2016) WSES Jerusalem guidelines for diagnosis and treatment of acute appendicitis. World JEmerg Surg 11:34

2. Gorter RR, Eker HH, Gorter-Stam MAW et al (2016) Diagnosis and management of acute appendicitis. EAES consensus development conference 2015. Surg Endosc 30:4668-4690 